\section{US pathology centre units will live on}

We wish to point out that several elements of the US Armed Forces Institute of Pathology (AFIP) have survived its closure and have been relocated within the Department of Defense (Nature 476, 270-272; 2011).

These units include the Depleted Uranium and Imbedded Fragment Laboratory, the Molecular Laboratory, Telepathology, the Automated Central Tumor Registry, the Veterinary Pathology Program (including residency training), the Armed Forces Medical Examiner function, the Histotechnology Training Program, and the congressionally funded Combat Wound Initiative. The Department of Veterans' Affairs has assumed responsibility for the capabilities of the Biophysical Research Laboratory.

The Department of Defense is working to make the vast collection of the former AFIP Tissue Repository (now part of the Joint Pathology Center) broadly available for research. At our request, the Institute of Medicine has convened a panel of national experts in biorepository management, medical informatics, medical ethics and pathology. The panel's task is to recommend the optimal and sustainable use of repository material; who should have access to it; technologies needed to utilize the repository; and ethical considerations over the use in research of material originally collected for clinical purposes.

Several institutes are collaborating in pathology translational research and in supporting key clinical-research initiatives and education efforts. These include the Uniformed Services University of the Health Sciences, the Joint Task Force National Capital Region Medical (and its subordinate units, the Joint Pathology Center and Walter Reed National Military Medical Center), other organizations in the Department of Defense, and Veterans' Affairs.

These collaborations

will be part of a new era of intergovernmental and public-private partnerships that will create vital research and clinical interactions. The celebrated history of AFIP and its importance to the broader research, clinical and academic communities provide the perfect foundation.

Thomas P. Baker Joint Pathology Center, Silver Spring, Maryland, USA.thomas.p.baker@us.army.mil John M. Mateczun Joint Task Force National Capital Region Medical, Bethesda, Maryland, USA.

Charles L. Rice Uniformed Services University of the Health Sciences, Bethesda, Maryland, USA.

\section{China's academic autocracy must go}

Many scientists in China share Nai-Xing Wang's dissatisfaction with the dominant role of journal impact factors in the country's scientific evaluation system (Nature 476, 253; 2011). But I contend that even an imperfect law is better than no law.

Replacing this rigid evaluation system with a more flexible one could send Chinese academia into chaos. Leaders of universities and research institutions could then establish their own evaluation systems, designing them to favour their particular interests. For example, a professor who is connected to a scientific journal might be tempted to rank papers published in that journal more highly when evaluating the performance of his or her university.

Chinese researchers should benefit from the strict implementation of impact-factor evaluation criteria. But the rewards for meeting these targets aren't always forthcoming. A good relationship with the few leading executives who control China's academia is also important, as it is for gaining access to the best scientific projects and for promotions.

The key task is therefore to eradicate this autocratic control. Researchers would then be able to concentrate solely on their work. Nai-Zhuo Zhao Northeast Normal University, Changchun, China.

naizhuo.zhao@gmail.com

\section{Review boards: vital to protect subjects}

On behalf of the Consortium of Independent Review Boards, a non-profit US organization for ethical review of clinical research and protection of participants, I object to your suggestion that a US government proposal to overhaul institutional review board (IRB) regulations would increase the use of commercial IRBs that have an "unsettling incentive to approve trials" (Nature 476, 125; 2011).

You imply that independent IRBs put research subjects at risk. However, all review boards are subject to a high level of federal regulation and inspection. Inspections by the US Food and Drug Administration involve thorough site visits and assessment of policies, procedures and records.

Independent IRBs protect research participants by reviewing studies conducted by private clinics and community and academic hospitals. Without such IRBs, patient access to promising experimental treatments would be curtailed and research would be unduly protracted.

Consortium members commit to a code of ethics requiring them to protect the IRB from economic influences when reviewing research, minimize 'IRB shopping' and promote ethical marketing. The consortium also requires members to be accredited by independent bodies such as the Association for the Accreditation of Human Research Protection Programs (see Nature 477, 280; 2011).

The consortium believes that the proposed overhaul of the regulations warrants careful review, and urges the research community to focus on identifying substantive measures that support the highest standards for protecting human subjects in clinical research. Cami Gearhart Consortium of Independent Review Boards, Washington DC, USA. www.consortiumofirb.org

\section{Make integrity key to recruitment}

Far from being a vague ideal, the complex and sensitive issue of maintaining integrity in science is a critical imperative. In my view, it would help to demand and monitor integrity in scientists and managers from the outset (Nature 476, 251, 262; 2011).

Most researchers know from their training that honesty is fundamental to scientific integrity. But some managers and agency officials can find themselves in difficult situations. A manager must cope with the competing pressures of supporting and protecting the scientists working on a project, ensuring the survival of the scientific institution and pleasing unforgiving political masters possibly all under public scrutiny. Even an honest manager might fear being undermined by a rival colleague or, worse, by a scientist who is cavalier about professional ethics.

The only way to achieve scientific integrity across the board is to ensure that personal and professional values (as well as knowledge and skills) are primary criteria for the employment of both scientists and managers. These values must be demonstrated and constantly monitored, not just presumed. Alfred P. Zarb Leura, New South Wales, Australia. zarbap@ozemail.com.au 